\title{
Young-Elderly Travellers as Potential Users and Actual Users of Internet with Mobile Devices during Trips
}

\author{
Niklas Eriksson and Susanna Fabricius \\ Arcada University of Applied Sciences \\ Department of Business Management and Analytics \\ Jan-Magnus Janssonin aukio 1, 00560 Helsinki, Finland \\ niklas.eriksson@arcada.fi, susanna.fabricius@arcada.fi
}

\begin{abstract}
The population is rapidly ageing in countries such as Finland. However, little research has been conducted to better understand older travellers' use of Internet and mobile devices. This qualitative study aims at exploring youngelderly (aged 60-75) travellers as potential users and actual users of the Internet with mobile device during trips. The results identify a range of possible drivers and barriers for the use of Internet with mobile devices and their impact on the travel experience during trips. The study also shows that there is a substantial number of young-elderly travellers that are quite advanced in their mobile usage behaviour.
\end{abstract}

Keywords: Older travellers, Travel experience, Mobile devices, Technology adoption and use, User behaviour, Digital services

\section{Introduction}

Many travellers are nowadays acting as their own travel agents and they build their own travel packages and trip itineraries [21]. Mobile technology such as tablet devices and smart phones is taking the digital development even further. A large scale survey by Hjalager and Jensen [10] confirms that many travellers want to be online before the trip, during the trip and after the trip. However, individuals adopt technology based innovations (services) very differently [20]. One consumer segment, which is becoming very relevant, is the so called young-elderly (aged 60 - 75) segment [1] [4]. Population ageing is faced by most developed countries. For example in Finland the proportion of persons aged 65 or over in the population is estimated to rise from the present $18 \%$ to $26 \%$ by 2030 and to $28 \%$ by 2060 [25]. Even globally the population aged 60 or over is the fastest growing [29]. However, only little research has been conducted on the influence of Internet in the older tourist market [17] [30]. On the other hand an ever growing number of older people in countries such as Finland are Internet users. This appears from Statistics Finland's survey on use of information and communications technology. In 2013, the upper age limit of the survey sample was raised from 74 to 89 years [26]. The number of mobile devices has also quickly increased in the oldest age groups. In fact, the adoption rates of smart phones for people aged $60-75$ have passed 
$50 \%$ in Finland [4]. Once older adults join the digital world it tends to become an integral part of their lives [23].

Based on the fact that individuals adopt and use online services differently, the lack of research of older tourists' online behaviour and the increased use of Internet and mobile devices in this segment, it seems necessary and timely to investigate youngelderly travellers' online behaviour. This study will focus on exploring young-elderly travellers as potential users and actual users of Internet with mobile devices during trips.

\section{$2 \quad$ Literature}

\subsection{Online Activities and Older Travellers}

Before the trip travellers are generally focused on assessing the destination and planning transportation and accommodation, whereas in the during trip settings the search strategies of tourists are primarily focused on planning the venue on-site, such as activities to undertake [22]. Seniors research the Internet for travel information in different ways, e.g. through Google searches and by visiting travel sites [27]. However, according to the same authors seniors rarely use social networks such as Facebook and Twitter for travel planning, but many of them have entered an 'e-buyer' era, where they actually buy travel products online. Generally speaking social media is growing in importance as an influence on the online travel information search process [34]. For example travel sites such as Tripadvisor provide global platforms for rating travel services and sharing experiences. Tourists often want to recall memories and share them with others with e.g. photos and stories both during the trip and after the trip [28]. Nowadays numerous travellers come pre-loaded with apps and content for mobile use. Mobile services have the potential to support tourists in different stages of the trip and mobile devices are used for many online travel activities, e.g. search, book and reflect [7]. Many travellers have developed new routines in during trip settings due to the use of a smart phone. These new routines include for example finding information about the travel, book tickets and taking photos and sharing with others immediately [33].In fact using a personal mobile device for online purposes during trips is seen by many travellers as important [10]. Minazzi and Mauri [14] point out that the use of mobile devices and applications affect the travel experience in different stages of the traveller life cycle. On the other hand Pesonen et al. [17] found that Finnish seniors seldom use their mobile devices to connect to the Internet during trips. Others have also emphasized that we should be careful to overestimate the extent of travellers' use of mobile devices [11]. However, neither should we underestimate the number of technology savvy older travellers. Research conducted by Reisenwitz et al. [19] shows that seniors are online more hours and more frequently and feel more comfortable online. Niemelä-Nyrhinen [15] concluded that elderly (baby boomers) in Finland have, generally speaking, a low level of technology anxiety.

\subsection{Drivers and Barriers of Technology Use and Impact on Travel Experience}

Several models have been developed to better understand individuals' adoption and use of information technology. One of the most widely used models is the technology 
acceptance model (TAM) by Davis [6] which is based on the theory of reason action (TRA) by Fishbein et al. [8] with routes in psychology theories and its extension theory of planned behavior (TPB) by Ajzen [2]. Other often used models in technology adoption research are the diffusion of innovations theories (DOI) by Rogers [20], the unified theory for the acceptance and use of technology (UTAUT) by Venkatech et al. [31] and the unified theory for consumers' acceptance and use of information technology (UTAUT2) by Venkatech et al. [32]. These models have been applied to explain the acceptance of different types of technologies by older adults [5]. Mallenius et al. [12] found through the lens of UTAUT, that Expected benefit, Perceived security, Usability, Anxiety, Training, Guidance, Price barriers and Social influence are relevant when investigating elderly individuals' mobile device and service use in Finland.

It should be noted that when investigating adoption and use of technology it is very important to distinguish between potential users and actual users. Gerpott [9] found that innovation-based attributes explained mobile Internet acceptance better for actual users than for potential users. Recent studies have also emphasized the need for research on the impact of technology on the tourist experience among those who actually uses their mobile devices [16]. The same authors concluded that emotional responses, missed opportunities, monetary burden and behavioural consequences may lead to negative travel experience effects. In fact, Wang et al. [33] emphasized that not only drivers of smart phone use in travel should be examined in order to understand its impact on travel experience, but also barriers (resistance) to use. Experiences of technology while travelling may also induce anxieties and tensions, due to technology addiction [18].

\section{$3 \quad$ Methodology}

We conducted a semi-structured qualitative study with 14 pensioners within the age group $60-75$ to better understand young-elderly travelers as potential users and as actual users of the Internet with mobile devices during trips. The sample was drawn from a Swedish speaking pensioners' association in Helsinki Finland. In order to gain as broad as possible view of the target segment both low-proficiency users of the Internet and high-proficiency users of the Internet were selected in collaboration with the association. Also low frequency travelers and high frequency travelers were selected. The selection of the sample can hence be described as purposive [13]. All informants were strangers to the two interviewers and the interviews lasted for about an hour on average. The interviews were conducted in spring 2015 and according to ethical standards and confidentiality by providing advance information to the informant regarding the purpose of the study, as well as about who will have access to the data and confidentiality guidelines of the project.

An instrument was developed to guide the interviews. The questions analyzed in this study are listed below:

- Describe how you mostly search for information during your trips

- Do you use the Internet with your smart phone and/or tablet device during your trips? 
- What do you do with them? Which device do you prefer?

- Is there a difference in how you use the device abroad compared to your use in Finland?

- Why do you use the Internet with your smart phone and/or tablet device during your trips?

- Why do you not use the Internet with your smart phone and/or tablet device during your trips?

- Do you see that that the use of the Internet with a smart phone and/or tablet device during your trips impacts your travel experience?

- How do you share your travel experiences during your trips?

The questions are flexible in character which allowed us to account for individual differences and take the advantage of the iterative nature of interviewing [13]. The interviews were conducted in Swedish and voice recorded. Direct citations have been translated to English by the authors.

The sample may be biased towards people with a higher educational degree and a larger household income than the average Finnish $60-75$ aged individuals (cf. [17]). On the other, hand our purpose was to select as diverse informants as possible based on travel frequency and online proficiency and not based on educational and economic situation. From table 1 we can see that the informants have a wide distribution in perceived online proficiency, annual travel frequency and numbers of trips during the past three years. Furthermore we are not aiming at generalizing results but to explore possible drivers and barriers for the potential use and actual use of the Internet with mobile devices during trips.

\section{$4 \quad$ Results}

According to the theory discussed and based on the results we have divided the sample into potential users and actual users of the Internet with mobile devices in during trip settings. Five informants (F1, F2, M7, F11 and M13) belong to potential users and nine (F3, F4, M5, M6, M8, F9, F10, M12 and F14) belong to actual users. With the theoretical discussion on technology use and impact on travel experience in mind we analysed the results of the two groups.

\subsection{Potential users}

The results are sub-divided into themes that represent the drivers and barriers of potential users of the Internet with mobile devices during trips. 
Table 1. Interview informants

\begin{tabular}{|c|c|c|c|c|c|c|c|c|c|c|c|c|c|c|}
\hline 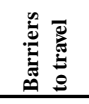 & 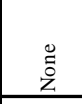 & 号 & \begin{tabular}{l} 
总 \\
\multirow{2}{*}{}
\end{tabular} & 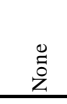 & $\begin{array}{l}\overline{5} \\
\overline{0} \\
\text { 㟧 }\end{array}$ & \begin{tabular}{l} 
总 \\
\multirow{2}{*}{}
\end{tabular} & : & 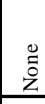 & $\begin{array}{l}\text { 言 } \\
\text { 言 } \\
\text { 恶 }\end{array}$ & \begin{tabular}{|l|} 
产 \\
至 \\
\end{tabular} & \begin{tabular}{|l|} 
产 \\
焉 \\
\end{tabular} & 总 & $\begin{array}{l}\text { 竞 } \\
\text { 全 } \\
\end{array}$ & $\begin{array}{l}\text { 琂 } \\
\text { 言 } \\
\end{array}$ \\
\hline 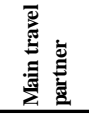 & 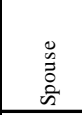 & $\mid$ & 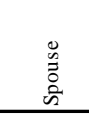 & 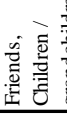 & 童 & 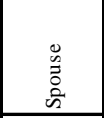 & 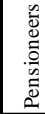 & \begin{tabular}{|l} 
\\
$\frac{\tilde{g}}{\alpha}$
\end{tabular} & 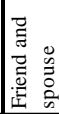 & 总 & \begin{tabular}{|l|} 
\\
$\frac{0}{\alpha}$ \\
\multirow{2}{*}{}
\end{tabular} & 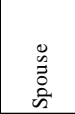 & 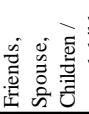 & 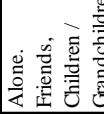 \\
\hline 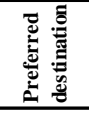 & 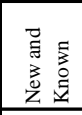 & 竞 & 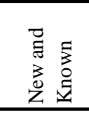 & 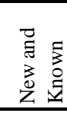 & $\frac{z}{\underline{z}}$ & $\begin{array}{l}\text { 言 } \\
\text { 总 } \\
\end{array}$ & \begin{tabular}{|l} 
\\
产 \\
\end{tabular} & 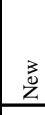 & 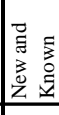 & \begin{tabular}{|l|}
\multirow{z}{z}{} \\
\multirow{2}{*}{} \\
\end{tabular} & \begin{tabular}{|l|} 
\\
\\
$\vdots$ \\
\end{tabular} & $\begin{array}{l}\text { 章 } \\
\end{array}$ & $\frac{3}{\underline{3}}$ & 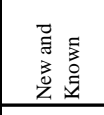 \\
\hline & 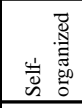 & 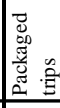 & 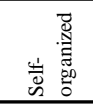 & 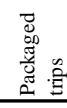 & $\begin{array}{ll}0 \\
\\
0 \\
0\end{array}$ & 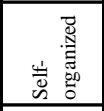 & 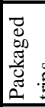 & $-\pi$ & 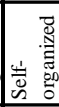 & 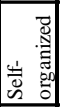 & 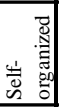 & 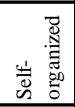 & 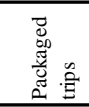 & 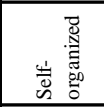 \\
\hline 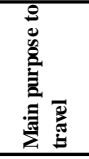 & 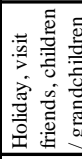 & 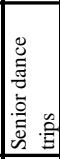 & 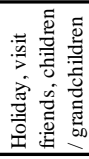 & 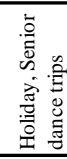 & 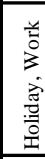 & 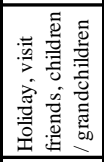 & 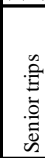 & 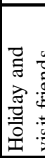 & 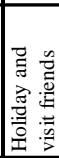 & 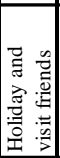 & 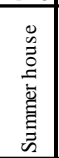 & 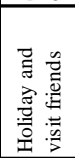 & $\begin{array}{l}\text { 苞 } \\
\text { 垔 }\end{array}$ & 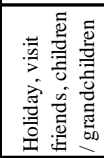 \\
\hline 毵言 & 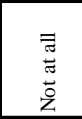 & 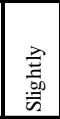 & 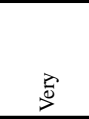 & $\begin{array}{l}\frac{5}{5} \\
\frac{5}{2} \\
\end{array}$ & : & $\stackrel{8}{D}$ & 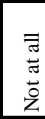 & 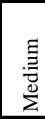 & \begin{tabular}{|l|}
$\frac{2}{0}$ \\
产 \\
童 \\
\end{tabular} & $\stackrel{2}{2}$ & 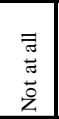 & 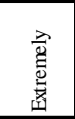 & $\stackrel{2}{2}$ & 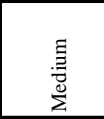 \\
\hline 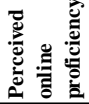 & ڤั & 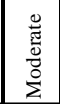 & 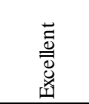 & 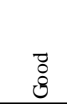 & $\frac{\frac{2}{2}}{\frac{\mathrm{g}}{8}}$ & $\begin{array}{l}\frac{0}{\frac{9}{5}} \\
\frac{y_{0}^{\circ}}{2}\end{array}$ & \begin{tabular}{|l}
7 \\
8 \\
\end{tabular} & 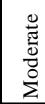 & 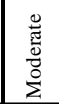 & \begin{tabular}{|l|}
$\frac{0}{0}$ \\
$\frac{0}{0}$ \\
$\frac{3}{2}$ \\
\end{tabular} & 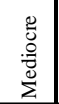 & $\begin{array}{l}\text { 䓂 } \\
\frac{\overline{\bar{g}}}{8} \\
\text { 啇 }\end{array}$ & 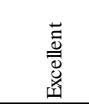 & 总 \\
\hline 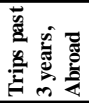 & $m$ & $\cong$ & $\simeq$ & $\alpha$ & $=$ & $\cong$ & $m$ & 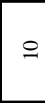 & $r$ & $m$ & - & r & + & 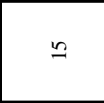 \\
\hline 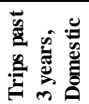 & $\infty$ & $\underline{n}$ & $\cong$ & - & + & $\cong$ & + & in & + & 0 & 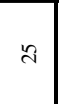 & $\cong$ & $\sim$ & 0 \\
\hline 焉 & 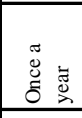 & 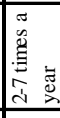 & 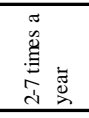 & 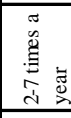 & 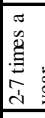 & 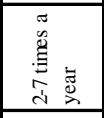 & E. & 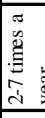 & 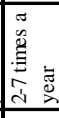 & 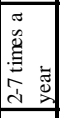 & 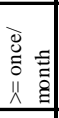 & 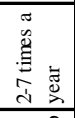 & 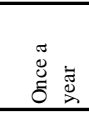 & 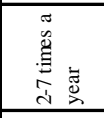 \\
\hline 焉 & 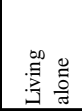 & 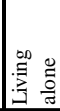 & 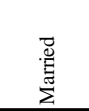 & 言 & 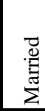 & 离 & 总 & 胞。 & $\begin{array}{l}\frac{\widetilde{d}}{\bar{E}} \\
\frac{\sqrt{5}}{2}\end{array}$ & 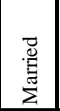 & 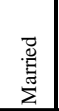 & 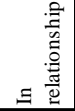 & 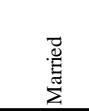 & 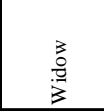 \\
\hline 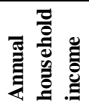 & : & $\begin{array}{l}8 \\
\text { did }\end{array}$ & $\begin{array}{l}\text { ष्. } \\
\text { \& }\end{array}$ & & $\dot{\sigma}$ & $\begin{array}{l}\stackrel{8}{8} \\
\stackrel{8}{-1}\end{array}$ & ষ্. & $\stackrel{8}{.8}$ & 兽 & $\begin{array}{l} \\
\\
\\
\end{array}$ & \begin{tabular}{|c|} 
\\
8 \\
6 \\
\end{tabular} & $\underset{8}{8}$ & $\begin{array}{l}8 \\
0 \\
0\end{array}$ & 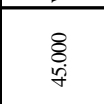 \\
\hline 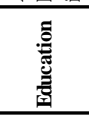 & 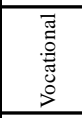 & $\overline{\underline{\sigma}}$ & 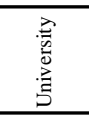 & 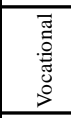 & 产 & 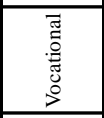 & 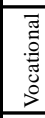 & 产 & 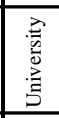 & 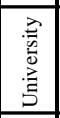 & 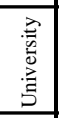 & 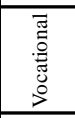 & 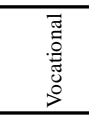 & 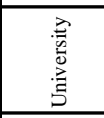 \\
\hline 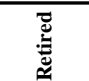 & ర్సీ & 品 & 윰 & . & . & ठ্ণ & छ్ & 売 & 売 & 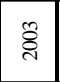 & $\underset{\pi}{8}$ & ڤ్ন & 党 & క్స \\
\hline $\bar{~}$ & $n$ & 8 & $\bar{F}$ & 8 & S & ल & 吉 & 8 & 8 & $\approx$ & 8 & 8 & 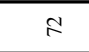 & $F$ \\
\hline 离 & $\frac{0}{20}$ & 焉 & 遌 & 迹 & $\Sigma$ & $\frac{0}{\frac{\omega}{\pi}}$ & $\frac{\pi}{2 \pi}$ & $\frac{\pi}{\tilde{\pi}}$ & 递 & 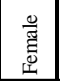 & 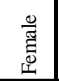 & $\frac{0}{\tilde{g}}$ & $\sum_{\frac{\pi}{\pi}}^{\frac{0}{\pi}}$ & 迹 \\
\hline 妾 & I & 포 & 番 & 吉 & $\sum$ & $\stackrel{\circ}{2}$ & E & \begin{tabular}{|l|}
$\infty$ \\
$\Sigma$
\end{tabular} & 现 & 焉 & $\bar{I}$ & $\frac{\tilde{\Sigma}}{\Sigma}$ & $\frac{m}{\Sigma}$ & $\frac{\vec{t}}{ \pm}$ \\
\hline
\end{tabular}




\section{Expected added-value}

F2, M7 and F11 said that they see themselves using a mobile device, either smart phone or tablet device, during the trip in the near future. They all three see potential added value by using a mobile device during a trip, e.g. fast and convenient access to information, locating themselves on a map. In fact two of them (F11, M7) had already purchased a smart phone, but had not taken it into use. F11 said that the smart phone was purchased specifically for the needs of an upcoming trip

"I need to be able to locate myself on a map during my trip to Spain and therefore I have purchased a smart phone, but I have not taken it into use yet." (F11)

F1 on the other hand found it more challenging to perceive the travel experience enhancement of using smart phones, rather she finds them making people anti-social and distracted from the world around them.

"I cannot really see the purpose of people sitting in trains, busses etc. with their heads down .... Phones should be used only as phones ..." (F1)

M13 said he owns an old smart phone that he does not use, because it is slow and he does not really need it.

\section{Travel style and partner}

F1 and F2 see that their travel style influences their behaviours and that they form different roles with their travel partners during the trip. In fact, because their travel partner uses a mobile device they do not need to use one themselves.

"I mostly travel together with my sister... My sister is searching for information with a mobile device during trips so I don't really need to. " (F2)

"When we were in Stockholm my friend used her tablet device to find information on where to go and what to do... I do the talking with locals and she looks for information." (F1)

M7, F1 and F2 mostly go on packaged trips (see table 1). M7 sees that because he mostly takes part in organised senior-trips he does not really need a mobile device to arrange things during the trip. F2 on the other hand sees herself using a personal mobile device when she goes on trips without her sister.

“I probably need to start using one, as I cannot go on every trip with my sister." (F2)

\section{Knowledge and guidance}

Four of the informants (F1, F2, M7 and F11) expressed that technology in general can be struggling and frustrating to use. They admit that their personal technology skills are limited but that it can be hard for them to admit to others that they have problems using technology appliances. M7 said that he has a smart phone waiting for him, but he has not taken it into use. The reason is that he needs help in learning how to use it.

"I have been struggling to make the effort to get it up and running. I should ask help from my daughter so that I will be able to use it." (M7) 


\begin{abstract}
Anxiety
Anxiety towards technology does not seem to be a hindrance for F2, F11 and M13 to become actual mobile device users during trips, as they are looking for and booking travel on the Internet in the pre-trip stage. F11 is mostly purchasing routine trips on the Internet (train, ferry and bus tickets) and F2 and M13 have purchased flights, accommodation and travel packages online. M7 is using the Internet for finding travel information but he has not tried to purchase travel online. M7 feels more comfortable using traditional travel agents to make trip arrangements and prefers to go on packaged senior-trips, rather than to organize them himself online.
\end{abstract}

"I call the local travel agent to make trip arrangements... I can trust them as I have used them a lot before." (M7)

F1 concluded that she is trying to stay away from technology appliances and even her children have told her to stay away from technology. She even considered her-self lucky to be out of working life due to so much now being computer-based and she could not cope with that. Her only point of contact with the Internet is if someone wants her to check some information on a web-site.

\title{
4.2 Actual users
}

The results are sub-divided into themes that represent actual drivers and barriers of using the Internet with mobile devices during trips and their impact on travel experience.

\section{Added-value}

The informants use Internet with mobile devices during trips to find local sights, check reviews, weather and opening hours, find the shortest routes to places etc. Map services are the most widely used mobile services. The informants find the mobile device convenient and time-saving as they have instant access to information in any situation. F14 said that she is very attached to her iPhone and F9 that it feels like she has inside information about local places as she can check other travelers' experiences online with her mobile device. This kind of information was not available to her before or it was not easily accessible. However, it can also have a negative effect on the travel experience as it may passivate travelers from asking locals etc., making travelers actually missing out on experiences.

"...on the other hand mobile devices may passivate us to ask locals, as we start to believe that we already know everything about the place. " (F9)

In fact, M8 sometimes intentionally leaves his mobile device out and asks e.g. the hotel reception for local tips as they may have some really valuable knowledge to offer. According to M8 visiting the online top rated attractions may not give him the authentic travel experience he is looking for. Also digital map services may be found too efficient.

"Nowadays we do not get lost and stumble into interesting things like we used to do." (F3) 
The informants also kill or fill time in transportation by using their mobile devices. Only one informant (M12) mentioned that he uploads pictures with his mobile device in social media during trips. All other informants prefer to share their experiences after the trip. However, social media is not extensively used for sharing travel experiences after the trip either, primarily due to unwillingness to share private information.

Mobile devices also give the informants a feeling of safety, that the traveler can be reached and that they can reach travel partners and people at home (e.g. SMS, e-mail, WhatsApp, Facebook). On the other hand the awareness of things at home may also increase tensions during the trip (see technology tensions below).

\section{Travel style}

All nine informants reported that they mainly organize their trips themselves, rather than taking part in packaged trips (see table 1). They find the mobile device is a key tool for their style of traveling and experiencing things. The following two narratives represent their responses well.

"I want to organize things myself and then the mobile device comes in handy." (M8)

"I can imagine that if I would take part in an organized trip I would not really need my mobile device as everything then is organized." (F9)

Some of them even feel reluctant to take part in organized senior-trips. It may even be hard for them to see or they don't want to see themselves as seniors by definition.

"That sounds a little bit boring... I think that is for people who cannot travel in another way....it is good that they are arranged but that is not for me." (M8)

\section{Usability}

All nine informants found mobile devices to be mostly easy to use and bring along while travelling. Most of them bring both a tablet device and a smartphone with them on the trip. However, there are different situations for using these two devices to enhance the travel experience. The tablet device is mostly used at the hotel (F3, F10, M5). For example M12 uses the tablet device at the hotel e.g. in the morning to plan what to see, while he carries his smart phone while wandering around during the day. The smartphone is smaller and therefore easier to carry around.

"I don't think I need to bring my tablet device on my next trip as we are going to backpack... the smartphone is easier to carry along." (F10)

On the other hand M5 and F3 find the tablet device easier to use due to its screen size. Aging may cause changes in visual capacity and other restrictions for self-arrangements.

"The tablet device is more convenient and sharper, I can see better with it." (M5)

One other hand F14 concluded that she does not need a tablet device as on her iPhone she can e.g. re-size the text. 
Many stated that mobile devices are their primary information and communication channel during trips nowadays. In fact, mobile devices have to some extent replaced e.g. traditional brochures, paper maps and sending postcards. As M6 stated

"They are so versatile, one can do anything with them; take pictures, search for information, communicate..." (M6)

On the other hand F9 finds it important to have a backup plan e.g. paper map just in case something goes wrong with the mobile device. She feels that she cannot totally rely on her mobile devices. Many of M6s friends find it very nice if he sends them a traditional postcard as no one else is doing that nowadays.

\section{Monetary burden}

Informants said that roaming charges abroad are a problem and depending on the destination they worry about them. For example M12 and F14 have partly guarded themselves from roaming charges in the Nordic and Baltic countries by purchasing a subscription that allows for data transfer at the same price as in Finland. The following three narratives represent well the informants' worries about the potential monetary burden.

"I have to turn off some updates on my phone while travelling abroad.” (F10)

"It is better to be careful with the use abroad so that I don't have a big bill waiting for me at home." (F4)

"When I went to the US I had to set the device in flight mode due to terrible roamingcosts.” (F3)

\section{Tensions}

Both M8 and M12 say that it is very important that the hotel has a proper Wifi, it can even be decisive of whether they will stay at the hotel or not. M12 feels that he will complain if the Wifi is not working properly or if it is over-charged.

"... I remember once in Sweden they over-charged for the Wifi, then I posted a complaint about that." (M12)

M12 brings his mobile devices everywhere, except perhaps to the beach. M12 also admits that he is addicted to his devices.

"I have to admit that I'm addicted .... I feel half naked if I forget my phone." (M12)

Some of them also admit that it can be frustrating to be in network-dead zones, being unable to connect to the Internet. Informants also mentioned that mobile devices make them not really getting away from home (they read what is going in the news at home, read their e-mails, Facebook updates etc.).

"One should turn off the phone...there is not really a need to be online all the time... " (F10) 


\section{$5 \quad$ Discussion and conclusions}

First we studied the potential users (five informants) of the Internet with mobile devices during trips. We found a range of possible drivers and barriers, but we sub-divided the results according to four thematic factors influencing the potential use; expected addedvalue, travel style and partner, knowledge and guidance and anxiety. Generally speaking there was a consensus in this group that there is added-value to use the Internet with mobile devices during a trip. Neither did technology anxiety seem to be a great hurdle, except for one informant. In fact, usage barriers seemed to be more related to their style of traveling, their personal knowledge of using technology and availability of support by e.g. a younger family member. This is in line with previous research that some guidance may be needed to push the adoption and use of mobile device and services by elderly [12]. It may, however, be hard for this group to admit that they need help with technology appliances, which may constitute a greater barrier than being anxious about learning new technologies.

Second we studied the actual users (nine informants) of the Internet with mobile devices during trips. Also in this group we identified a range of possible drivers and barriers, but we sub-divided the results according to five thematic factors influencing the actual use and the travel experience; added-value, travel style, usability, monetary burden and tensions. The findings indicate there is a consensus in the group that there definitely is added-value in using the Internet and mobile devices during trips and it affects the travel experience positively. However, sharing their travel experiences with their mobile devices in social media is not widely used. They also see that they may passivate as travelers and that they may miss out on authentic travel experiences due to their use of mobile devices. Usability is generally not a problem, rather they reflect about which mobile device(s) to use and bring along. Two of them preferred a tablet device (larger screen) due to restrictions in visual capacity. They all found that a mobile device is handy for their most common style of traveling, self-organized trips. The usage barriers and the negative effects on travel experience are to be found in technology tensions that are caused by e.g. roaming costs in international travel contexts, poor Wifi and dead zones. However, these usage barriers or travel experience barriers are also typical for younger travelers [7] [16]. One of the informants even admitted that he is addicted to his mobile devices.

To sum it up, in this study the conventional description of seniors as technology anxious and highly reluctant to use technology ([15] [17]) was not widely recognized. Rather this study shows that there is a substantial number of young-elderly travelers' who are quite advanced in using the Internet with mobile devices during trips. In fact, some informants' behavior rather resembles addiction towards mobile devices than anxiousness towards them [18]. However, we should not generalize the results to a total population due to the explorative and qualitative research approach and the purposive sample selection. Quantitative research could empirically verify the results on a larger scale and test dependency between the variables suggested in this study. The analysis of the interview results could also be extended and interpreted to a theoretical model. Limiting the sample to $60-75$ year old seniors, here referred to as young-elderly, ob- 
viously decreases the importance of health and physical capacity issues to use technology. A 75+ sample may indeed give us very different results [23]. On the other hand, according to Mallenius et al. [12], age is not really the key, rather it is the functional capacity that matters when determining how mobile devices and services are perceived. When studying the behavior of elderly consumers, not only the chronological age should be discussed, but also the cognitive age (how old one "feels" to be) [3] [24]. In this study a substantial number of the informants could not see themselves as seniors by definition.

\section{References}

1. Allmér, H., Råberg, M.: Young-elderly and digital use. In: IRIS36 - 36th Information Systems Research Seminar in Scandinavia: "Digital living", Oslo, Norway (2013)

2. Ajzen, I.: The Theory of Planned Behavior. J. Organizational Behavior and Human Decision Processes. 50, 179-211 (1991)

3. Barak, B., Leon, G. S.: Cognitive Age: a Non chronological Age Variable. NA - Advances in Consumer Research. 08, 602-606 (1981)

4. Carlsson, C., Walden, P., Vogel, D., Merne, M.: Young Elderly - Progressive Market for Digital Service?. In: Panel discussion in the 27th Bled eConference, Bled, Slovenia (2014)

5. Chen, K.., Chan, A. H. S.: A review of technology acceptance by older adults. Gerontechnology. 10(1), 1-12 (2011)

6. Davis, F. D.: Perceived Usefulness, Perceived Ease of Use and User Acceptance of Information Technology. J. MIS Q. 13 (3), 319--340 (1989)

7. Eriksson, N. (2014). User categories of mobile travel services. J. of Hospitality and Tourism Technology, 5(1): 17-30 (2014)

8. Fishbein, M., Ajzen, I.: Belief, attitude, intention and behavior: an introduction to theory and research. Reading, MA Addison-Wesley (1975)

9. Gerpott, T.J.: Attribute perceptions as factors explaining Mobile Internet acceptance of cellular customers in Germany - An empirical study comparing actual and potential adopters with distinct categories of access appliances. Expert Systems with Applications, 38(3): 2148-2162 (2011)

10. Hjalager, A-M., Jensen, J.M.: A typology of travellers based on their propensity to go online before, during and after the trip. In: Fuchs, M., Ricci, F., Cantoni, L. (eds.), Information and Communication Technologies in Tourism 2012, Helsingborg, Sweden, pp. 96107. Springer, Wien (2012)

11. Douglas, A., Lubbe, B.: Mobile Devices as a Tourism Distribution Channel. In: Tussyadiah I., Xiang, Z. (eds.), Information and Communication Technologies in Tourism 2014, Dublin, Irland, pp. 855-867. Springer, Wien (2014)

12. Mallenius S., Rossi M., Tuunainen V.K.: Factors affecting the adoption and use of mobile devices and services by elderly people -results from a pilot study. In: $6^{\text {th }}$ Annual Global Mobility Roundtable. Los Angeles (2007)

13. McGehee, N., G.: Interview Techniques. In: Dwyer, L., Gill, A., Seetaram, N. (eds.) Handbook of Research Methods in Tourism. Edward Elgar, Cheltenham, UK (2012)

14. Minazzi, R., Mauri, A., G.: Mobile Technologies Effects on Travel Behaviours and Experiences: A Preliminary Analysis. In: Tussyadiah I., Inversini, A. (eds.) Information and Communication Technologies in Tourism 2015, Lugano, Switzerland, pp. 507- 522. Springer, Switzerland (2015) 
15. Niemelä-Nyrhinen, J.: Baby boom consumers and technology: Shooting down stereotypes. Journal of Consumer Marketing, 24(5): 305-312 (2007)

16. Neuhofer, B., Buhalis, B., Ladkin, A.: Technology as a Catalyst of Change: Enablers and Barriers of the Tourist Experience and Their Consequences. In: Tussyadiah I., Inversini, A. (eds.) Information and Communication Technologies in Tourism 2015, Lugano, Switzerland, pp. 789- 802. Springer, Switzerland (2015)

17. Pesonen. J., Komppula, R., Riihinen, A.: Senior Travellers as Users of Online Travel. In: I. Tussyadiah I., Inversini, A. (eds.) Information and Communication Technologies in Tourism 2015, Lugano, Switzerland, pp. 831 - 846. Springer, Switzerland (2015)

18. Paris, C.M., Berger, E.A., Rubin, S., Casson, M.: Disconnected and Unplugged: Experiences of Technology Induced Anxieties and Tensions While Traveling. In: Tussyadiah I., Inversini, A. (eds.) Information and Communication Technologies in Tourism 2015, Lugano, Switzerland, pp. 803- 816. Springer, Switzerland (2015)

19. Reisenwitz, T., Iyer, R., Kuhlmeier, D., Eastman, J.: The elderly's internet usage: an updated look. J. of Consumer Marketing, 24 (7): 406-418 (2007)

20. Rogers, E., M.: Diffusion of Innovations. Free Press, 5th edition, New York (2003)

21. Sigala, M.: Measuring customer value in online collaborative trip planning processes. Marketing Intelligence \& Planning, 28(4): 418-443 (2010)

22. Sirakaya E., Woodside, A. G.: Building and Testing Theories of Decision Making by Travellers. Tourism Management, 26(6): 815-832 (2005)

23. Smith, A.: Older Adults and Technology Use. PewResearchCentre (2014), Retrieved April 15, 2015 from http://www.pewinternet.org/2014/04/03/older-adults-and-technology-use/

24. Sudbury L., Simcock, P.: A multivariate segmentation model of senior consumers, J. of Consumer Marketing. 26(4): 251-262 (2009)

25. Statistics Finland: Population projection, Retrieved March 5, 2015 from http://tilastokeskus.fi/til/vaenn/2012/vaenn_2012_2012-09-28_tie_001_en.html

26. Statistics Finland: Over one-quarter of persons aged 75 to 89 use the Internet. Retrieved January 15, 2015, from http://www.stat.fi/til/sutivi/2013/sutivi_2013_2013-1107_tie_001_en.html

27. Thébault, M., Picard, P., Ouedraogo, A.: Seniors and Tourism: An International exploratory study on the use of the Internet for researching recreational information use. International Business Research, 6(3): 22 (2013)

28. Tussyadiah, I.P., Fesenmaier, D. R.: Mediating Tourists Experiences-Access to Places via Shared Videos. Annals of Tourism Research, 36(1): 24-40 (2009)

29. United Nations: World Population ageing (2013), Retrieved March 5, 2014 from http://esa.un.org/unpd/wpp/Documentation/pdf/WPP2012_HIGHLIGHTS.pdf

30. Vigolo, V., Confente, I.: Older Tourists: An Exploratory Study on online Behavior. In: Tussyadiah I., Xiang, Z. (eds.), Information and Communication Technologies in Tourism 2014, Dublin, Irland, pp. 439 - 449. Springer, Wien (2014)

31. Venkatesh, V., Morris, M. G., Davis, G. B., Davis, F. D.: User acceptance of information technology: toward a unified view. J. MIS Q. 27(3), 425--478 (2003)

32. Venkatesh, V., Tong, J.Y.L., Xu, X.: Consumer Acceptance And Use Of Information Technology: Extending The Unified Theory Of Acceptance And Use Of Technology. J. MIS Q. 36(1): 157-178 (2012)

33. Wang, D., Xiang, Z., Fesenmaier, D.R.: Smartphone Use in Everyday Life and Travel. Journal of Travel Research published online 19 May 2014

34. Xiang, Z., Gretzel, U: Role of social media in online travel information search. Tourism Management, 31(2): 179-188 (2010) 